\title{
Two-dimensional Neutral Coulomb Gas
}

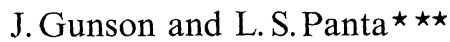 \\ Department of Mathematical Physics, University of Birmingham, \\ Birmingham B15 2TT, England
}

\begin{abstract}
Accurate bounds for the classical canonical partition function of the two-dimensional Coulomb gas interacting through the Coulomb potential $-q_{i} q_{j} \log r_{i j}$ are calculated (valid for all $T>T_{c}$ ). The existence of the thermodynamic limit is proved.
\end{abstract}

In a recent paper Deutsch and Lavaud [1] speculated about the thermodynamic limit for the two-component classical neutral Coulomb gas in twodimensions, i.e. the existence of finite thermodynamic functions per particle in the limit $N \rightarrow \infty, V \rightarrow \infty$ with $\frac{N}{V}$ bounded.

Their estimates were not accurate enough to prove the existence of the thermodynamic limit. Recently Fröhlich [12] has solved the problem using euclidean quantum field techniques (Gaussian integration).

The objective of this paper is to obtain stronger bounds for the canonical partition functions of the Coulomb gas and then prove the existence of the thermodynamic limit using well established statistical mechanics techniques.

The general scheme of the paper is, by sections, as follows:

1. Definitions. The partition function, free energy per particle, etc. are defined and the problem of the thermodynamic limit precisely stated.

2. Upper bound for classical partition function.

3. Basic inequality for classical systems. An inequality relating the canonical partition function for domain $\Omega$ to the partition function for two sub-domain $s \Omega^{\prime}$ and $\Omega^{\prime \prime}$ is obtained.

4. Limit for cubes. A standard sequence $\Gamma_{k}$ of cubic domains, each double the size of its predecessor is introduced. The existence of the limiting free energy is established for this sequence by showing (essentially) that the free energy per particle is monotonically increasing in $k$ and recalling the upper bound (Section 2).

* On leave from Universidade Federal da Paraiba (Brazil)

$\star \star$ Supported in part by Conselho Nacional de Desenvolvimento Cientifico e Tecnologico, Brazil and the British Council 
5. Properties of the free energy. It is shown from the basic inequality that the limiting free energy is a convex function of the specific volume $v=1 / \varrho$, whence it follows that $f(v)$ is continuous and differentiable. This establishes the existence of the limiting pressure and its monotonicity in $v$.

\section{Definitions}

We consider a 2-dimensional Euclidean space with position vectors $r$. A domain of this space will be denoted by $\Omega$ and will have a volume $V=V(\Omega)$.

A mechanical system of $2 N$ classical charged particles, $N$ positive and $N$ negative will be specified by its Hamiltonian,

$$
\mathscr{H}_{2 N}=\mathscr{H}_{2 N}\left(p_{i}, r_{i}\right)=T_{2 N}+U_{2 N}
$$

where the kinetic energy is

$$
T_{2 N}=\sum_{i=1}^{2 N} p_{i}^{2} / 2 m
$$

and the potential energy

$$
U_{2 N}=-\sum_{1 \leqq i<j \leqq 2 N} q_{i} q_{j} \log \left|r_{i}-r_{j}\right| ; \quad q_{i}\left\{\begin{array}{l}
+q i=1, \ldots, N \\
-q i=N+1, \ldots, 2 N .
\end{array}\right.
$$

Here $p_{i}, r_{i}$, and $q_{i}$ are the momentum, position vectors and charge of the $i^{\text {th }}$ particle.

Denoting $\beta=1 / k_{B} T$ and $\Lambda=\left(h^{2} / 2 \pi m k_{B} T\right)^{\frac{1}{2}}$ the canonical partition function for the neutral Coulomb gas is

$$
Z(\beta, 2 N, \Omega)=\Lambda^{-4 N} Q(\beta, 2 N, \Omega)
$$

where

$$
Q(\beta, 2 N, \Omega)=(N !)^{-2} \int \ldots \int \prod_{i=1}^{2 N} d^{2} r_{i} \exp \left[-\beta U_{2 N}\right] .
$$

The canonical free energy is defined through the formulas

$$
Z(\beta, 2 N, \Omega)=e^{2 N f(\beta, 2 N, \Omega)}=e^{V g(\beta, \varrho, \Omega)}
$$

and

$$
f(\beta, 2 N, \Omega)=F_{2 N} / 2 N k_{B} T=v g(\beta, \varrho, \Omega)
$$

where the density $\varrho$ and specific volume $v$ are defined by

$$
\varrho=2 N / V(\Omega)=v^{-1} .
$$

Given a sequence of domains $\Omega_{k}(k=0,1, \ldots)$ with $V\left(\Omega_{k}\right) \rightarrow \infty$ the thermodynamic limit may now be defined, when it exists, by

$$
\lim _{k \rightarrow \infty} g\left(\beta, \varrho, \Omega_{k}\right)=g(\beta, \varrho)=v^{-1} f(\beta, v)
$$

where the limit is taken at fixed $\varrho$. 
If the limiting functions are differentiable, the canonical pressure is defined by $\beta p=(\partial f / \partial v)=g-\varrho(\partial g / \partial \varrho)$

\section{Upper Bound for Classical Partition Function}

We can write the canonical partition function in the form

$$
Z(\beta, 2 N, \Omega)=(N !)^{-2}\left(V / \Lambda^{2}\right)^{2 N} V^{\frac{-\beta q^{2} N}{2}} Q_{2 N}^{*}
$$

where

$$
Q_{2 N}^{*}=\int_{\left|S_{i}\right| \leqq 1} \ldots \int_{i=1}^{2 N} d^{2} S_{i} \exp \left(\beta \sum_{i<j} q_{i} q_{j} \log \left|S_{i}-S_{j}\right|\right)
$$

and we used the scaling procedure

$$
S_{i}=r_{i} V^{-\frac{1}{2}}
$$

to separate out the volume dependence.

\subsection{Electrostatic Inequality}

A basic tool is an inequality whose source is closely related to the fact that nonoverlapping spherically symmetric charge distributions exert forces on each other as if their total charge was concentrated in their centre.

Let $l_{1}, l_{2}, \ldots, l_{n}$ be rings with respective centers $x_{1}, x_{2}, \ldots, x_{n} \in R^{2}$ and arbitrary radii $r_{1}, \mathrm{r}_{2}, \ldots, r_{n}$. Suppose there is a uniform layer of charge on each $l_{i}$. Let its linear density be $q_{i} / 2 \pi r_{i}$, where $q_{i}$ may have either sign. This produces a continuous potential in $R^{2}$ given by

$$
\phi(x)=-\sum_{i=1}^{2 N}\left(q_{i} / 2 \pi r_{i}\right) \int_{l_{i}} \log \left|t_{i}-x\right| d l_{i}
$$

The total electrostatic energy is

$$
(4 \pi)^{-1} \int_{R^{2}}|\nabla \phi|^{2} d^{2} x=-\frac{1}{2} \sum_{i=1}^{2 N} \sum_{j=1}^{2 N}\left(q_{i} / 2 \pi r_{i}\right)\left(q_{j} / 2 \pi r_{j}\right) \iint_{l_{i} l_{j}} \log \left|t_{i}-t_{j}\right| d l_{i} d l_{j}
$$

where we used the first Green's identity and the fact that we have a neutral system to show that the boundary contribution vanishes. For non-overlapping charge distributions and recognizing that the diagonal terms $(i=j)$ are just the self energies we obtain

$$
(4 \pi)^{-1} \int_{R^{2}}|\nabla \phi|^{2} d^{2} x=-\frac{1}{2} \sum_{\substack{i, j=1 \\ i \neq j}}^{2 N} q_{i} q_{j} \log \left|\mathrm{x}_{i}-\mathrm{x}_{j}\right|-\frac{1}{2} \sum_{i=1}^{2 N} \mathrm{q}_{i}{ }^{2} \log \mathrm{r}_{i} \geqq 0
$$


or

$$
-\frac{1}{2} \sum_{\substack{i, j=1 \\ i \neq j}}^{2 N} q_{i} q_{j} \log \left|x_{i}-x_{j}\right| \geqq \frac{1}{2} \sum_{j=1}^{2 N} q_{i}^{2} \log r_{i}
$$

which is the electrostatic inequality for non-overlapping charges in two dimensions.

An accurate upper bound may be given to the integral (2.2) by the following procedures.

1. Remove from the region of integration all points in which two or more of the separations $\left|S_{i}-S_{j}\right|$ coincide. This set of points has measure zero. In the remaining domain of integration $\Omega$, each point $S_{i}$ has a unique nearest neighbour $S_{F(i)}$, where $F(i)$ depends on the particular configuration of points $\left(S_{1}, \ldots, S_{2 N}\right)$ chosen.

2. The $4 N$-dimensional domain $\Omega$ is partitioned into disjoint subsets $\{U\}$, where each $U$ consists of all configurations with the same nearest neighbour function $i \rightarrow F(i)$. Thus there is one subdomain $U$ for each nearest neighbour function on the index set $\{1, \ldots, 2 N\}$. The union of the over all possible nearest neighbour function is just $\Omega$.

3. We construct a uniform bound for the integrations over the subdomains $U$ and an estimate of the number of such subdomains to give an overall estimate of the integral (2.2).

\subsection{Upper Bound for (2.2)}

By making use of the electrostatic inequality (2.3) we can write

$$
\left.Q_{2 N}^{*} \leqq \int_{\left|S_{i}\right| \leqq 1} \ldots \int \prod_{i=1}^{2 N} d^{2} S_{i} \exp \left(\beta / 2 \sum_{i=1}^{2 N} q^{2} \log \frac{1}{2} \operatorname{Min}_{i \neq j}\left|S_{i}-S_{j}\right|\right)\right)
$$

consider now the integral

$$
I_{U}=\int \ldots \int \prod_{i=1}^{2 N} d^{2} S_{i} \exp \left(-\beta / 2 \sum_{i=1}^{2 N} q^{2} \log \left(\frac{1}{2}\left|S_{i}-S_{F(i)}\right|\right)\right)
$$

where $U$ is a subdomain associated with the nearest neighbour function $i \rightarrow F(i)$, and $\left|S_{i}-S_{F(i)}\right|$ represent the distance between $i$ and its nearest neighbour. The function $F$ is conveniently represented by a directed graph (digraph) and is of the form:
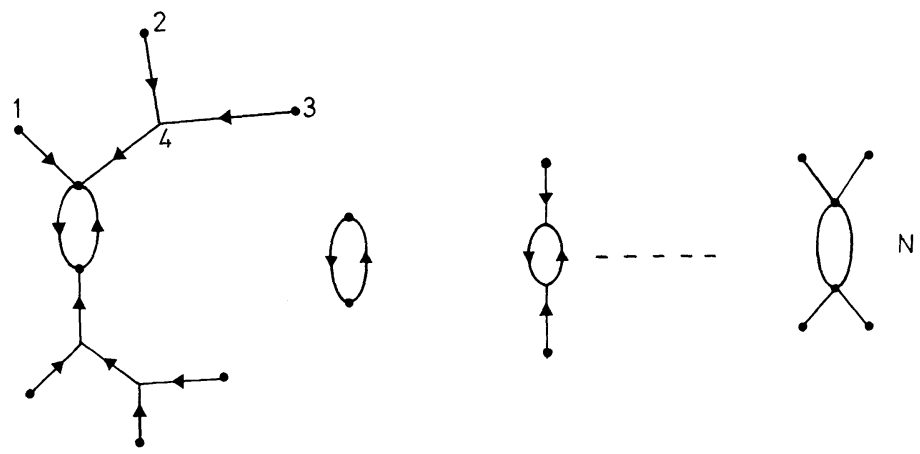

i.e. a graph of 1 to $N$ connected components each of which is composed of a cycle of two lines, together with trees attached to the two nodes on the cycle. The nearest neighbour condition forbids cycles with three or more lines. Each distinct labelling 
of the $2 N$ nodes gives a different nearest neighbour function. The geometrical restrictions of two-dimensional space prohibit any point being the nearest neighbour to more than five other points.

Case a). The graph has only one component (i.e. is connected). We may suppose, without loss of generality, that $S_{2 N}$ and $S_{2 N-1}$ are the points on the cycle. Then we may perform the following change of variables in the integral $(2.5)$

$$
\begin{aligned}
& S_{1} \ldots \rightarrow u_{1} \ldots=\frac{1}{2}\left(S_{1}-S_{F(1)}\right) \\
& \dot{S_{j}} \ldots \rightarrow u_{j} \ldots=\frac{1}{2}\left(\dot{S_{j}}-\dot{S}\right. \\
& \left.\dot{S_{2 N}} \ldots \rightarrow u_{2 N-1}\right) \\
& \dot{S}_{2 N} \rightarrow \frac{1}{2}\left(\dot{S_{2 N-1}}-\dot{S}_{2 N}\right) \\
& S_{2 N}=\frac{1}{2} S_{2 N} .
\end{aligned}
$$

The Jacobian of the transformation is $2^{2 N}$. Hence

$$
I_{U} \leqq 2^{2 N} \int_{D} \ldots \int_{D} \frac{d^{2} u_{1} \ldots d^{2} u_{2 N}}{\left(\left|u_{1}\right| \ldots\left|u_{2 N-2}\right|\left|u_{2 N-1}\right|^{2}\right)^{\frac{1}{2} \beta q^{2}}}
$$

where $D$ is a suitably enlarged domain for the $U$-integration variables, chosen so that the integral becomes easier to evaluate. We take the domain

$$
D=\left\{\sum_{i=1}^{2 N-1}\left|u_{i}\right|^{2} \leqq 4,\left|u_{2 N}\right| \leqq \frac{1}{2}\right\} \text {. }
$$

Because $\left|S_{i}\right| \leqq 1$ and $\frac{1}{2} S_{2 N}=u_{2 N}$ then $\left|u_{2 N}\right| \leqq \frac{1}{2}$. Also $\left|u_{i}\right|$ is $\frac{1}{2}$ the nearest neighbour distance from the $i^{\text {th }}$ point. Hence the set of discs

$$
D_{i}=\left\{\begin{array}{l}
\text { set of points in } R^{2} \text { at a distance from } S_{i} \text { not exceeding }\left|u_{i}\right|, \\
i=1,2, \ldots, 2 N-1
\end{array}\right\}
$$

form a non-overlapping set of total area

$$
\pi \sum_{i=1}^{2 N-1}\left|u_{i}\right|^{2}
$$

confined into a disc of radius at most 2 in the $s$-plane. Hence

$$
\pi \sum_{i=1}^{2 N-1}\left|u_{i}\right|^{2} \leqq \pi 2^{2}
$$

and, in terms of the original $s$-variables, $D$ includes $U$.

Using a standard integral [3, page 258] we get

$$
I_{U} \leqq(2 \pi)^{2 N} 4^{-1} \cdot 2^{2(2 N-2)\left(1-\frac{1}{4} \beta q^{2}\right)} \cdot 2^{\left(1-\frac{1}{2} \beta q^{2}\right)} \cdot \frac{\Gamma\left(1-\frac{1}{4} \beta q^{2}\right)^{2 N-2} \Gamma\left(1-\frac{1}{2} \beta q^{2}\right)}{\Gamma\left[(2 N-2)\left(1-\frac{1}{4} \beta q^{2}\right)+\left(1-\frac{1}{2} \beta q^{2}\right)+1\right]} .
$$

Case $b$ ). The graph has $k$ components. Likewise, for nearest neighbour functions with $k$ connected components we have an estimate

$$
\begin{aligned}
I_{u} \leqq & (2 \pi)^{2 N} 4^{-k} \cdot 2^{2(2 N-2 k)\left(1-\frac{1}{4} \beta q^{2}\right)} \cdot 2^{2 k\left(1-\frac{1}{2} \beta q^{2}\right)} \\
& \cdot \frac{\Gamma\left(1-\frac{1}{4} \beta q^{2}\right)^{2 N-2 k} \Gamma\left(1-\frac{1}{2} \beta q^{2}\right)^{k}}{\Gamma\left[(2 N-2 k)\left(1-\frac{1}{4} \beta q^{2}\right)+k\left(1-\frac{1}{2} \beta q^{2}\right)+1\right]} \\
\equiv & Y\left(1-\frac{1}{4} \beta q^{2}\right)^{2 N-2 k} X\left(1-\frac{1}{2} \beta q^{2}\right)^{k} / \Gamma\left[(2 N-k)-(N / 2) \beta q^{2}+1\right]
\end{aligned}
$$

where $Y(u)$ and $X(u)$ are $N$ and $k$-independent functions. 
We now need an estimate of the number of nearest neighbour functions with $k$ components. So we enumerate the nearest neighbour regions $U$. This can be carried out using standard techniques for graphical enumeration [4, pp. 8, 20,69].

The exponential generating function for the number of labelled connected functional digraphs with cycle of order 2 is

$$
C(t)=\frac{z^{2}}{2}=\sum_{n=1}^{\infty}(n-1) n^{n-2} t^{n} / n !
$$

and the exponential generating function for the number of labelled functional digraphs with exactly $K^{\prime}$ components is

$$
\frac{1}{K !} C(t)^{K^{\prime}}=\frac{1}{K !}\left(\frac{z^{2}}{2}\right)^{K^{\prime}}
$$

By comparison with formula (A.4) of appendix and setting $K=2 K^{\prime}$, we have

$$
\frac{1}{K^{\prime} !} C(t)^{K}=\sum_{n=1}^{\infty} \frac{(n-1) ! n^{n-2 K} t^{n}}{2^{K-1}(K-1) !(n-2 K) ! n !}
$$

so the number of graphs of the above type with precisely $K$-components and $2 \mathrm{~N}$ nodes is

$$
\frac{2(2 N-1) !(2 N)^{2 N-2 K}}{2^{K}(K-1) !(2 N-2 K) !} \leqq \frac{\Gamma(2 N+1)(2 N)^{2 N-2 K}}{2^{K} \Gamma(K+1) \Gamma(2 N-2 K+1)} .
$$

Thus, multiplying (2.7) by (2.8) and summing the result over $K$ we obtain the following upper bound for $Q_{2 N}^{*}$

$$
\begin{aligned}
Q_{2 N}^{*} \leqq & (2 N)^{\left(\beta q^{2} N\right) / 2} \sum_{K=1}^{N} \frac{\Gamma(2 N+1) \exp (2 N)}{\Gamma(K+1) \Gamma(2 N-K+1)}\left[Y\left(1-\left(\beta q^{2}\right) / 4\right)\right]^{2 N-K} \\
& \cdot\left[X\left(1-\left(\beta q^{2}\right) / 2\right)\right]^{K}(2 Y)^{-K} \leqq(2 N)^{\left(\beta q^{2} N\right) / 2} \exp (2 N)[Y+(X / 2 Y)]^{2 N}
\end{aligned}
$$

using Newton's binomial formula.

With the help of definition (1.2) and formulas (2.1) and (2.9) we obtain immediately for the free energy per particle.

$$
f(\beta, 2 N, \Omega) \leqq 1-\log \frac{\Lambda^{2} \varrho}{2}+\frac{\beta q^{2}}{4} \log \varrho+\log \left[e Y+\frac{e X}{2 Y}\right]
$$

It should be noted that (2.10) does not make sense for $T \leqq T_{c}=q^{2} / 2 k_{B}$. The function $Y$ diverges for $T=T_{c}$. As Knorr [10] has shown the partition function does no longer exist for $T \leqq T_{c}$ and the system collapses in configuration space.

\section{Basic Inequality for Classical Systems}

Consider two sub-domains $\Omega^{\prime}$ and $\Omega^{\prime \prime}$ with non overlapping volumes [7] but lying within the volume of a domain $\Omega$. We will derive an inequality between the partition functions for $\Omega, \Omega^{\prime}$, and $\Omega^{\prime \prime}$. Firstly, remembering that the integrand of the configurational integral (1.1) is positive, reduce the domain of integration of each $r_{i}$ 
to $\Omega^{\prime}+\Omega^{\prime \prime}$. Next split up the total $4 N$-fold domain of integration in all possible ways into combinations of $\Omega^{\prime}$ and $\Omega^{\prime \prime}$ keeping always an equal number of positive and negative charges in $\Omega^{\prime}$ and $\Omega^{\prime \prime}$. This yields the inequality

$$
\begin{aligned}
Z(\beta, 4 N, \Omega) \geqq & \frac{\Lambda^{-8 N}}{(2 N !)^{2}} \sum_{2 N^{\prime}+2 N^{\prime \prime}=4 N} \frac{(2 N !)^{2}}{\left(N^{\prime} !\right)^{2}\left(N^{\prime \prime} !\right)^{2}} \iint_{\Omega^{\prime}} \prod_{i=1}^{2 N^{\prime}} d^{2} r_{i} \iint_{\Omega^{\prime \prime}} \prod_{i=1}^{2 N^{\prime \prime}} d^{2} r_{i} \\
& \cdot \exp \left[-\beta U_{2 N^{\prime}}-\beta U_{2 N^{\prime \prime}}-\beta U_{2 N^{\prime}, 2 N^{\prime \prime}}\right] .
\end{aligned}
$$

Since all terms in the sum are positive the inequality remains true if we keep only one.

Introducing the measure

$$
d \mu=\frac{\exp \left[\beta U_{2 N^{\prime}}+\beta U_{2 N^{\prime \prime}}\right] \prod_{i=1}^{2 N^{\prime}} d^{2} r_{i} \prod_{i=1}^{2 N^{\prime \prime}} d^{2} r_{i}}{\iint_{\Omega^{\prime}} \prod_{i=1}^{2 N^{\prime}} d^{2} r_{i} \iint_{\Omega^{\prime \prime}} \prod_{i=1}^{2 N^{\prime \prime}} d^{2} r_{i} \exp \beta\left[U_{2 N^{\prime}}+U_{2 N^{\prime \prime}}\right]} .
$$

We can write (3.1) in the form

$$
Z(\beta, 4 N, \Omega) \geqq Z\left(\beta, 2 N^{\prime}, \Omega^{\prime}\right) Z\left(\beta, 2 N^{\prime \prime}, \Omega^{\prime \prime}\right) \int d \mu \exp \beta U_{2 N^{\prime}, 2 N^{\prime \prime}}
$$

and using Jensen's inequality we obtain

$$
Z(\beta, 4 N, \Omega) \geqq Z\left(\beta, 2 N^{\prime}, \Omega^{\prime}\right) Z\left(\beta, 2 N^{\prime \prime}, \Omega^{\prime \prime}\right) \exp \beta \int d \mu U_{2 N^{\prime}, 2 N^{\prime \prime}} .
$$

If we take the term in (3.1) such that the charges in $\Omega^{\prime}$ or $\Omega^{\prime \prime}$ are conjugated to the ones in (3.3) we get the inequality.

$$
Z(\beta, 4 N, \Omega) \geqq Z\left(\beta, 2 N^{\prime}, \Omega^{\prime}\right) Z\left(\beta, 2 N^{\prime \prime}, \Omega^{\prime \prime}\right) \exp \left(-\beta \int d \mu U_{2 N^{\prime}, 2 N^{\prime \prime}}\right)
$$

therefore from (3.3) and (3.4) we obtain the basic inequality

$$
Z(\beta, 4 N, \Omega) \geqq Z\left(\beta, 2 N^{\prime}, \Omega^{\prime}\right) Z\left(\beta, 2 N^{\prime \prime}, \Omega^{\prime \prime}\right) .
$$

This charge conjugation trick is due to Griffiths [13]. If this symmetry is not present the more general method of Lieb and Lebowitz [14] can still be used.

\section{Limit for Standard Cubes}

Up to this point we have used spherical domains. To simplify calculations we now introduce a standard sequence of cubic domains $[7] \Gamma_{k}(k=0,1, \ldots)$ which could be used to compare with more general sequences of domains. This brings no difficulty because for an upper bound one can always embed a cube in a sphere.

Let the edge of the cube $\Gamma_{k}$ be

$$
d_{k}=2^{k} d_{0}, \quad d_{k+1}=2 d_{k}
$$

so that the volume is

$$
V_{k}=V\left(\Gamma_{k}\right)=d_{k}^{v}=2^{v k} d_{0}^{v} \quad(v=\text { dimension }) .
$$


In the following we shall fill the standard cubes with particles observing the convention: each cube will have charge neutrality.

For brevity write for the free energy density

$$
g\left(\varrho, \Gamma_{k}\right)=g_{k}(\varrho) \text {. }
$$

Then on applying the basic inequality (3.5) with $\frac{1}{2} 2^{v}$ cubes $\Gamma$ at density $\varrho^{\prime}$ and $\frac{1}{2} 2^{v}$ at density $\varrho^{\prime \prime}$ we find

$$
g_{k+1}(\varrho) \geqq \frac{1}{2} g_{k}\left(\varrho^{\prime}\right)+\frac{1}{2} g_{k}\left(\varrho^{\prime \prime}\right)
$$

with

$$
\varrho=\frac{1}{2} \varrho^{\prime}+\frac{1}{2} \varrho^{\prime \prime} .
$$

For $\varrho^{\prime}=\varrho^{\prime \prime}=\varrho(4.1)$ yields the single inequality.

$$
g_{k+1}(\varrho) \geqq g_{k}(\varrho) \text {. }
$$

Thus $g_{k}$ is a monotonic increasing sequence bounded through (2.10) and (1.2). So the limit exists.

\section{Properties of the Free Energy}

The previous section establishes the existence of the limiting free energy for a sequence of cubes for all densities at which the partition function of a sufficiently large cube is non zero.

As we have seen before, the volume dependence of $Z(\beta, 2 N, \Omega)$ may be separated out with the aid of the scaling procedure. Consequently it is not difficult to determine the following dependence, on the density, for the free energy

$$
g(\beta, \varrho, \Omega)=\varrho\left\{\left(\frac{\beta q^{2}}{4}-1\right) \log \varrho+\log \left[\frac{2 N\left(Q_{2 N}^{*}\right)^{\frac{1}{2} N}}{\Lambda^{2}(N !)^{1 / N}(2 N)^{\frac{\beta q^{2}}{4}}}\right]\right\}
$$

so, for $\left(\beta q^{2}\right) / 2<1$, we have the sequence $g\left(\beta, \varrho, \Omega_{k}\right)$ as convex functions of $\varrho$.

\subsection{Limit for General Domains}

By using the basic inequality to compare the partition functions for a general sequence of domains $\Omega_{k}$ with those for the standard cubes contained within $\Omega_{k}$ and containing $\Omega_{k}$ it is possible to show that the free energy is independent of shape.

\subsection{Grand Canonical Ensemble}

Assuming the existence of the limiting canonical free energy the limiting grand canonical potential can be shown to exist and to yield equivalent results. 


\subsection{Field Theory}

The sine-Gordon theory is defined by the formal Lagrangian density [11]

$$
\mathscr{L}=\frac{1}{2} \partial_{\mu} \phi \partial^{\mu} \phi+\left(\alpha_{0} / \beta^{2}\right) \cos \beta \phi+\gamma_{0} \text {. }
$$

It is possible, using Euclidean Field Theory [12] to establish an isomorphism between the sine-Gordon theory and the Coulomb gas. This isomorphism allow us to use the bounds calculated above and prove the existence of the ground state energy density when the space cut off is removed.

\section{Appendix. Some Generating Functions}

\section{Lagrange Series [3, page 133]}

Let $f(z)$ and $\phi(z)$ be analytic inside and on the contour $C$ containing the point $a$ and such that

$$
|t \phi(z)|<|z-a| \text { on } C \text {. }
$$

Then

$$
\zeta=a+t \phi(\zeta)
$$

has one root $(\zeta)$ inside $C$. And for any $f$ as above

$$
f(\zeta)=f(a)+\left.\sum_{n=1}^{\infty} \frac{t^{n}}{n !}\left(\frac{d}{d r}\right)^{n-1}\left(f^{\prime}(r) \phi(r)^{n}\right)\right|_{r=a} .
$$

\section{Application}

1. Set $\phi(z)=e^{z}, a=0$ than the Equation (A.1) becomes $\zeta=t e^{\zeta}$ and the series (A.2) for $f=\zeta$,

$$
\zeta=\sum_{n=1}^{\infty} \frac{t^{n}}{n !}\left|\left(\frac{d}{d r}\right)^{n-1} e^{n r}\right|_{r=0}=\sum_{n=1}^{\infty} n^{n-1} \frac{t^{n}}{n !}
$$

this converges for $|t|<\frac{1}{e}$.

2. Let $f(z)=z^{K}$, then $\zeta=t e^{\zeta}$ gives

$$
\begin{aligned}
z^{K} & =\left.\sum_{n=1}^{\infty} \frac{t^{n}}{n !}\left(\frac{d}{d r}\right)^{n-1}\left(K r^{K-1} e^{n r}\right)\right|_{r=0} \\
& =\sum_{n=1}^{\infty} \frac{(n-1) !}{(n-K) !} K n^{n-K} \frac{t^{n}}{n !} .
\end{aligned}
$$

\section{References}

1. Deutsch,C., Lavaud,M.: Phys. Rev. A9, 2598 (1974)

2. Lenard, A.: Statistical mechanics and mathematical problems. Lecture Notes in Physics, Vol.20. Berlin-Heidelberg-New York: Springer 1973

3. Whittaker,E.D., Watson,G.N.: A course of modern analysis, 4th Ed. Cambridge 1952 
4. Harary,F., Palmer, E.: Graphical enumeration. New York-London: Academic Press 1973

5. Indritz, J.: Methods in analysis. New York: The MacMillan Company 1963

6. Mitrinovic,D.S. : Analytic inequalities. Berlin-Heidelberg-New York: Springer 1970

7. Fisher,M.E.: Arch. Rational Mech. Anal. 17, 377 (1964)

8. Simon, B.: The $P(\phi)_{2}$ Euclidean (quantum) field theory. Princeton: University Press 1974

9. Ruelle,D.: Statistical mechanics. New York: W. A.Benjamin Inc. (1969)

10. Knorr, G. : Phys. Letters 28A, 166 (1968)

11. Coleman,S.: Phys. Rev. D, 11, 2088 (1975)

12. Fröhlich,J.: International School of Mathematical Physics, 1975 at Erice, notes

13. Griffiths, R. B.: Phys. Rev. 176, 655 (1968)

14. Lieb,E., Lebowitz,J.: Advan. Math. 9, 316 (1972)

Communicated by G. Gallavotti

Received June 7, 1976 